\title{
Ocean circulation generated magnetic signals
}

\author{
C. Manoj ${ }^{1,2}$, A. Kuvshinov ${ }^{3,4}$, S. Maus ${ }^{1,5}$, and H. Lühr ${ }^{1}$ \\ ${ }^{1}$ GeoForchungsZentrum-Potsdam, Germany \\ ${ }^{2}$ National Geophysical Research Institute, Hyderabad, India \\ ${ }^{3}$ Danish National Space Center, Copenhagen, 2100, Denmark \\ ${ }^{4}$ Geoelectromagnetic Research Institute, Troitsk, Russia \\ ${ }^{5}$ NOAA's National Geophysical Data Center, Boulder, U.S.A.
}

(Received November 15, 2004; Revised March 29, 2005; Accepted April 21, 2005; Online published April 14, 2006)

\begin{abstract}
Conducting ocean water, as it flows through the Earth's magnetic field, generates secondary electric and magnetic fields. An assessment of the ocean-generated magnetic fields and their detectability may be of importance for geomagnetism and oceanography. Motivated by the clear identification of ocean tidal signatures in the CHAMP magnetic field data we estimate the ocean magnetic signals of steady flow using a global 3-D EM numerical solution. The required velocity data are from the ECCO ocean circulation experiment and alternatively from the OCCAM model for higher resolution. We assume an Earth's conductivity model with a surface thin shell of variable conductance with a realistic 1D mantle underneath. Simulations using both models predict an amplitude range of $\pm 2 \mathrm{nT}$ at $S$ warm altitude $(430 \mathrm{~km})$. However at sea level, the higher resolution simulation predicts a higher strength of the magnetic field, as compared to the ECCO simulation. Besides the expected signatures of the global circulation patterns, we find significant seasonal variability of ocean magnetic signals in the Indian and Western Pacific Oceans. Compared to seasonal variation, interannual variations produce weaker signals.
\end{abstract}

Key words: Ocean flow, geomagnetic field, satellite measurements.

\section{Introduction}

As the conducting water in the ocean moves in the ambient geomagnetic field of the Earth, it induces secondary electric and magnetic fields. This phenomenon is a case of motional induction, where the electrically charged ions in the sea water are deflected by the Lorentz force perpendicular to both the velocity and magnetic field vectors (Tyler et al., 2003). The spatial charge accumulation causes electric currents within the conducting water and bottom sediments. Based on the geometry of these currents, the ocean induced magnetic fields may be classified into "toroidal" and "poloidal". The toroidal components are caused by electric currents closing in the vertical plane and have an amplitude range of 10 to $100 \mathrm{nT}$ and are confined to the ocean and the upper crust. The poloidal components arise from the electrical currents closing in the horizontal plane and have a much smaller amplitude range (1 to $10 \mathrm{nT}$ ) at the sea level. However its large spatial decay scale allows the poloidal fields to reach outside the ocean to remote land and satellite based sensors. The poloidal magnetic field components, observed outside the ocean are mainly proportional to the depth integrated velocities (transport) of the ocean (Tyler et al., 1999). The possibility of identifying the ocean magnetic signals on land and satellite based magnetometer records have two implications. The ocean magnetic signals can give information about the major water transport

Copyright (c) The Society of Geomagnetism and Earth, Planetary and Space Sciences (SGEPSS); The Seismological Society of Japan; The Volcanological Society of Japan; The Geodetic Society of Japan; The Japanese Society for Planetary Sciences; TERRAPUB. across the oceans and their variability (Vivier et al., 2004). On the other hand, the non trivial contribution of ocean magnetic signals to the geomagnetic field necessitates accounting for them in geomagnetic field modeling (e.g. Maus et al., 2005).

Faraday (1832) was the first to recognize the motional induction in sea water. Larsen (1968) and Sanford (1971) were the modern day pioneers in the investigation of the ocean generated electric fields. The effect of the ocean dynamics, coastline and the electrical structure of the Earth on the induced electromagnetic fields were discussed by Chave (1983). Attempts to estimate ocean induced electric and/or magnetic fileds with realistic ocean circulation models have been made by Stephenson and Bryan (1992), Flosadóttir et al. (1997a,b), Tyler et al. (1997, 1999) and Palshin et al. (1999). Recently Vivier et al. (2004) correlated the variation in the simulated magnetic fields with the water transport across the Drake Passage. The predicted electric and/or magnetic fields were validated by undersea-cable voltage measurements (cf. Larsen and Sanford, 1985), land based electrical or magnetic field measurements (Junge, 1988; Maus and Kuvshinov, 2004), sea floor or sea surface magnetic field measurements (Lilley et al., 2004a,b) and satellite magnetic measurements (Tyler et al., 2003).

The use of satellite magnetic data is particularly promising, considering their global coverage, and the small spatial decay scale of the ocean magnetic signals. The ocean generated magnetic field has a typical strength of $1 \mathrm{nT}$ at CHAMP satellite altitude. To separate such signals from the observed magnetic signal (of a typical strength 50,000 
nT) is not a trivial task. However, with the advancement in the technology to measure and process the satellite magnetic data, it is now possible to identify the tidal magnetic signals in CHAMP data (Tyler et al., 2003). The low frequency magnetic signals produced by the quasi-stationary ocean circulations may be more difficult to separate as compared to tidal magnetic signals. However the Swarm mission, which intends to measure the Earth's magnetic field at an unprecedented precision promises to detect the ocean magnetic signals associated with ocean circulation. This paper details the result of the simulations carried out to assess the detectability of ocean magnetic signals with the Swarm mission. The primary objectives of the simulation were 1) To assess the large scale as well as small scale magnetic field effects of the ocean circulation at sea level and satellite altitude and 2) to examine their temporal variability. In addition, characterizing the ocean magnetic signals is an important first step in trying to identify the ocean magnetic signals in the satellite magnetic data. The first part of the paper discusses the forward computation, i.e. the electromagnetic numerical solution and the ocean circulation models used for the simulation. The results from the forward computation are discussed in the second part. The temporal variations of the ocean magnetic signals are treated in the last part of the paper.

\section{Forward Computation of Ocean Magnetic Sig- nals}

In order to calculate the magnetic fields due to global ocean steady flow, we have adopted the numerical solution which is described in Kuvshinov et al. $(2002,2005)$ and which has been already successfully applied for ocean tidal signals simulations (Maus and Kuvshinov, 2004; Kuvshinov and Olsen, 2005). The solution is based on a volume integral equation approach, which combines the modified iterative dissipative method (MIDM; Singer, 1995) with a conjugate gradients iteration. The solution allows simulating the electromagnetic (EM) fields, excited by arbitrary sources in three-dimensional (3-D) spherical models of electric conductivity. These models consist of a number of anomalies of 3-D conductivity, embedded in a host section of radially symmetric (1-D) conductivity. Within this approach Maxwell's equations in the frequency domain,

$$
\begin{gathered}
\nabla \times \mathbf{H}=\sigma \mathbf{E}+\mathbf{j}^{e x t}, \\
\nabla \times \mathbf{E}=i \omega \mu_{o} \mathbf{H},
\end{gathered}
$$

are reduced, in accordance with the MIDM, to a scattering equation of specific type (Pankratov et al., 1997)

$$
\chi(\mathbf{r})-\int_{V^{\bmod }} K\left(\mathbf{r}, \mathbf{r}^{\prime}\right) R\left(\mathbf{r}^{\prime}\right) \chi\left(\mathbf{r}^{\prime}\right) d v^{\prime}=\chi_{o}(\mathbf{r}),
$$

which is solved by the generalized bi-conjugate gradient method (Zhang, 1997). In the equations above $\mathbf{j}^{\text {ext }}$ is the exciting current, its time-harmonic dependency is $e^{-i \omega t}, \mu_{o}$ is the magnetic permeability of free space, $i=\sqrt{-1}, \omega=$ $2 \pi / T$ is the angular frequency, $T$ is the period of variations, $\sigma(r, \vartheta, \varphi)$ is the conductivity distribution in the model, $\vartheta, \varphi$ and $r$ are co-latitude, longitude and the distance from the
Earth's centre, respectively, $\mathbf{r}=(r, \vartheta, \varphi), \mathbf{r}^{\prime}=\left(r^{\prime}, \vartheta^{\prime}, \varphi^{\prime}\right)$, $V^{m o d}$ is the modelling region, and

$$
\begin{gathered}
R=\frac{\sigma-\sigma_{o}}{\sigma+\sigma_{o}} \\
K\left(\mathbf{r}, \mathbf{r}^{\prime}\right)=\delta\left(\mathbf{r}-\mathbf{r}^{\prime}\right) I+2 \sqrt{\sigma_{o}(r)} G_{o}^{e}\left(\mathbf{r}, \mathbf{r}^{\prime}\right) \sqrt{\sigma_{o}\left(r^{\prime}\right)} \\
\chi_{o}=\int_{V^{m o d}} K\left(\mathbf{r}, \mathbf{r}^{\prime}\right) \frac{\sqrt{\sigma_{o}}}{\sigma+\sigma_{o}} \mathbf{j}^{s}\left(\mathbf{r}^{\prime}\right) d v^{\prime} \\
\chi=\frac{1}{2 \sqrt{\sigma_{o}}}\left(\left(\sigma+\sigma_{o}\right) \mathbf{E}^{s}+\mathbf{j}^{s}\right) \\
\mathbf{j}^{s}=\left(\sigma-\sigma_{o}\right) \mathbf{E}^{o} \\
\mathbf{E}^{o}=\int_{V^{e x t}} G_{o}^{e}\left(\mathbf{r}, \mathbf{r}^{\prime}\right) \mathbf{j}^{e x t}\left(\mathbf{r}^{\prime}\right) d v^{\prime}
\end{gathered}
$$

Here $\delta\left(\mathbf{r}-\mathbf{r}^{\prime}\right)$ is Dirac's delta function, $I$ is the identity operator, $V^{e x t}$ is the volume occupied by the exciting current $\mathbf{j}^{\text {ext }}, \mathbf{E}^{s}=\mathbf{E}-\mathbf{E}^{o}$ is the scattered electric field, $G_{o}^{e}$ is the $3 \times 3$ dyadic Green's function of the 1-D reference conductivity $\sigma_{o}(r)$.

Once $\chi$ is determined from the solution of the scattering equation (3), the magnetic field, $\mathbf{H}$, at the observation points, $\mathbf{r} \in V^{o b s}$ (in our case $V^{o b s}$ is the surface of the Earth) is calculated as

$$
\mathbf{H}=\int_{V^{\text {ext }}} G_{o}^{h}\left(\mathbf{r}, \mathbf{r}^{\prime}\right) \mathbf{j}^{\text {ext }}\left(\mathbf{r}^{\prime}\right) d v^{\prime}+\int_{V^{\bmod }} G_{o}^{h}\left(\mathbf{r}, \mathbf{r}^{\prime}\right) \mathbf{j}^{q}\left(\mathbf{r}^{\prime}\right) d v^{\prime}
$$

with

$$
\begin{aligned}
\mathbf{j}^{q} & =\left(\sigma-\sigma_{o}\right)\left(\mathbf{E}^{o}+\mathbf{E}^{s}\right), \\
\mathbf{E}^{s} & =\frac{1}{\sigma+\sigma_{o}}\left(2 \sqrt{\sigma_{o}} \chi-\mathbf{j}^{s}\right) .
\end{aligned}
$$

Explicit expressions for the elements of $G_{o}^{e}$ and $G_{o}^{h}$ are given in the appendix of Kuvshinov et al. (2002). Having the magnetic field at the surface of the Earth one can recalculate the magnetic field at the satellite altitude, using a spherical decomposition of the magnetic fields calculated at the surface of the Earth.

As the present study focuses on the steady state ocean flow, the period of simulation is set to a large value. Since our 3-D conductivity model of the Earth consists of a surface spherical shell of $S(\theta, \varphi)$ underlain by a radially symmetric conductor, $\mathbf{j}^{\text {ext }}$ reduces to the sheet current density, $\mathbf{J}_{\tau}^{e x t}$, which is calculated as

$$
\mathbf{J}_{\tau}^{\text {ext }}=\sigma_{w}\left(\mathbf{U} \times \mathbf{e}_{r} B_{r}^{m}\right),
$$

where $\times$ denotes the vector product, $\sigma_{w}=3.2 \mathrm{~S} / \mathrm{m}$ is the mean sea water conductivity, $\mathbf{U}$ is the depth integrated velocity of global steady ocean flow, $\mathbf{e}_{r}$ is the outward unit vector and $B_{r}^{m}$ is the radial component of the main magnetic field derived from a model IGRF 2000. The shell conductance $S(\vartheta, \varphi)$ consists of contributions from the sea water and from sediments. The conductance of the sea water has been derived from the ocean salinity, temperature and pressure data given by World Ocean Atlas 2001 (www.nodc.noaa.gov), following the formulations by Fofonoff (1985). Conductance of the sediments is based on 
the global sediment thickness given by the $1^{\circ} \times 1^{\circ}$ map of Laske and Masters (1997) and calculated by a heuristic procedure similar to that described in Everett et al. (2003). Figure 1 presents the map of the surface shell conductance of $0.25^{\circ} \times 0.25^{\circ}$ resolution. For the underlying spherical conductor we choose a four-layer Earth model (similar to that described in Schmucker, 1985) instead of assuming an insulating mantle (cf. Vivier et al., 2004). It consists of a $100 \mathrm{~km}$ resistive lithosphere with $3000 \Omega \mathrm{m}$ followed by a moderately resistive first layer of $70 \Omega \mathrm{m}$ down to $500 \mathrm{~km}$, a second transition layer of $16 \Omega m$ from $500 \mathrm{~km}$ to 750 $\mathrm{km}$, and an inner uniform sphere of $0.42 \Omega \mathrm{m}$. Each simulation (with ECCO velocity data) took 1.2 hours CPU time on one processor of the type SunFire V8800 for a model discretization of $N_{\theta} \times N_{\phi}=180 \times 360$ and with an angular resolution of $1^{\circ} \times 1^{\circ}$. When simulating magnetic fields based on the OCCAM data, using a model discretization of $N_{\theta} \times N_{\phi}=720 \times 1440$ and with an angular resolution of $0.25^{\circ} \times 0.25^{\circ}$, it took 20 hours for the same processor.

\subsection{Ocean circulation models}

We obtained the velocity data for the forward computations from two ocean circulation models viz. ECCO (http://www.ecco-group.org) and OCCAM (http://www. soc.soton.ac.uk/JRD/OCCAM). The ECCO (Estimating the Circulation and Climate of the Ocean) model is based on the MIT general circulation model (Marshall et al., 1997). We use the data from the ECCO 1 model run (1992 to 2002). Using a model adjoint approach, the ECCO 1 model was forced to be consistent with the World Ocean Circulation Experiment (WOCE) observations. The model run was configured globally with a $1^{\circ} \times 1^{\circ}$ horizontal resolution, within $\pm 80^{\circ}$ latitudes and with 23 levels in the vertical. The horizontal components of the velocity data were interpolated to a common grid and were vertically integrated to obtain the transport data. The monthly transport grids from January 1992 to December 2002 were used to simulate the ocean magnetic signals. Additionally, the data from a high resolution ocean model OCCAM (Ocean Circulation and Climate Advance Modeling) were used to simulate the magnetic field effects of the ocean eddies. The model has 36 levels in the vertical starting with a thickness of $20 \mathrm{~m}$ at the surface and increasing to $250 \mathrm{~m}$ at depth. The spatial resolution of the model is $0.25^{\circ} \times 0.25^{\circ}$ (Webb et al., 1998). Archived OCCAM velocity data are available for every 15 days for most of the analysis period within the latitudinal limits $+66^{\circ}$ to $-77^{\circ}$. However, velocity data are not available for the North Atlantic Ocean region. For the Swarm simulations we used the average horizontal velocity fields from the 2985 and 3000 days of the OCCAM model run. Figure 2 shows the magnitude distribution of velocity data from both models. Basically, both models describe the major features of the ocean flow in a similar way. However ocean eddies are resolved only by the OCCAM model. For OCCAM data, the missing data for the North Atlantic Ocean region were filled with ECCO data.

\section{Results}

The radial components of the predicted magnetic field $\left(B_{r}\right)$ from ECCO and OCCAM data are presented in the Fig. 3. The dominant ocean induced magnetic signal is the radial component, $B_{r}$ and the horizontal components have about half of that strength. We do not plot other components, as all fields are poloidal, the horizontal fields do not contain additional information. At sea level (bottom panels), both predictions are within the amplitude range $\pm 10 \mathrm{nT}$ and look quite similar. However on closer inspection, high-intensity and short-wavelength features are more evident in OCCAM simulations. The maxima and minima of magnetic fields are $4 \mathrm{nT}$ and $-6 \mathrm{nT}$ for ECCO and $\pm 10 \mathrm{nT}$ for OCCAM simulations. The effect of ocean eddies is clearly visible in the Kuroshio Current region and along the Antarctic Circumpolar Current (ACC) region. These effects are by and large, absent in the ECCO simulations. Arguably, the smaller but intense flows resolved by the OCCAM model resulted in stronger magnetic signals at sea level. However, at satellite altitude $(430 \mathrm{~km})$ the predicted magnetic fields only contain information about the large scale features of the ocean circulation. Both predictions are within the amplitude range $\pm 2 \mathrm{nT}$. The magnetic fields from ECCO data have a slightly higher amplitude above the ACC in the Southern Indian Ocean (ECCO: $-1.5 \mathrm{nT}$ and OCCAM: $-1.2 \mathrm{nT}$ ) at satellite altitude. Both simulations are predominantly influenced by the ACC and Western boundary currents. The eastward flowing ACC results in two prominent anomalies to the east and west of the southern geomagnetic pole (located in the South Australian Ocean). The predicted amplitudes of the magnetic fields at satellite altitude are in agreement with the computations by Tyler et al. (1999).

\subsection{Magnetic field effect of ocean eddies}

A detailed map of the Kuroshio Current region is given in Fig. 4. The bottom panel shows the radial component of the predicted magnetic field at sea level, and the upper panel the magnetic field at satellite altitude. Both maps are superimposed on the velocity vectors from the OCCAM results. The induced magnetic fields due to eddies are evident at sea level, with a large amplitude range of $\pm 10 \mathrm{nT}$. These anomalies can be considered as a compounded effect of magnetic fields generated by individual eddies. As the ocean-continent boundary represents a large lateral contrast in conductance, the variation in sensitivity of the magnetic fields with respect to the conductance also must be taken into account. The one-to-one relationships between eddies and predicted anomalies are difficult to explain considering these complications. At satellite altitude the magnetic fields of individual eddies are not resolved (upper panel of Fig. 4). Here, the magnetic signatures of the Kuroshio Current exist as smooth elongated anomalies running parallel to the coast. This result stress the need to use a high resolution (and eddy resolving) ocean model while looking for the ocean signals in the magnetic records from the land observatories located near to coast.

\subsection{Variability of the ocean magnetic signals}

Ocean circulation is driven by winds on the surface and density differences due to varying water temperature and salinity. The depth integrated velocities used for our study are affected by the time variations in these forcing factors. While mean ocean magnetic signals may be difficult to remove from the crustal magnetic field $( \pm 20 \mathrm{nT}$ at an altitude of $400 \mathrm{~km}$ ), their temporal variation distinguishes them 

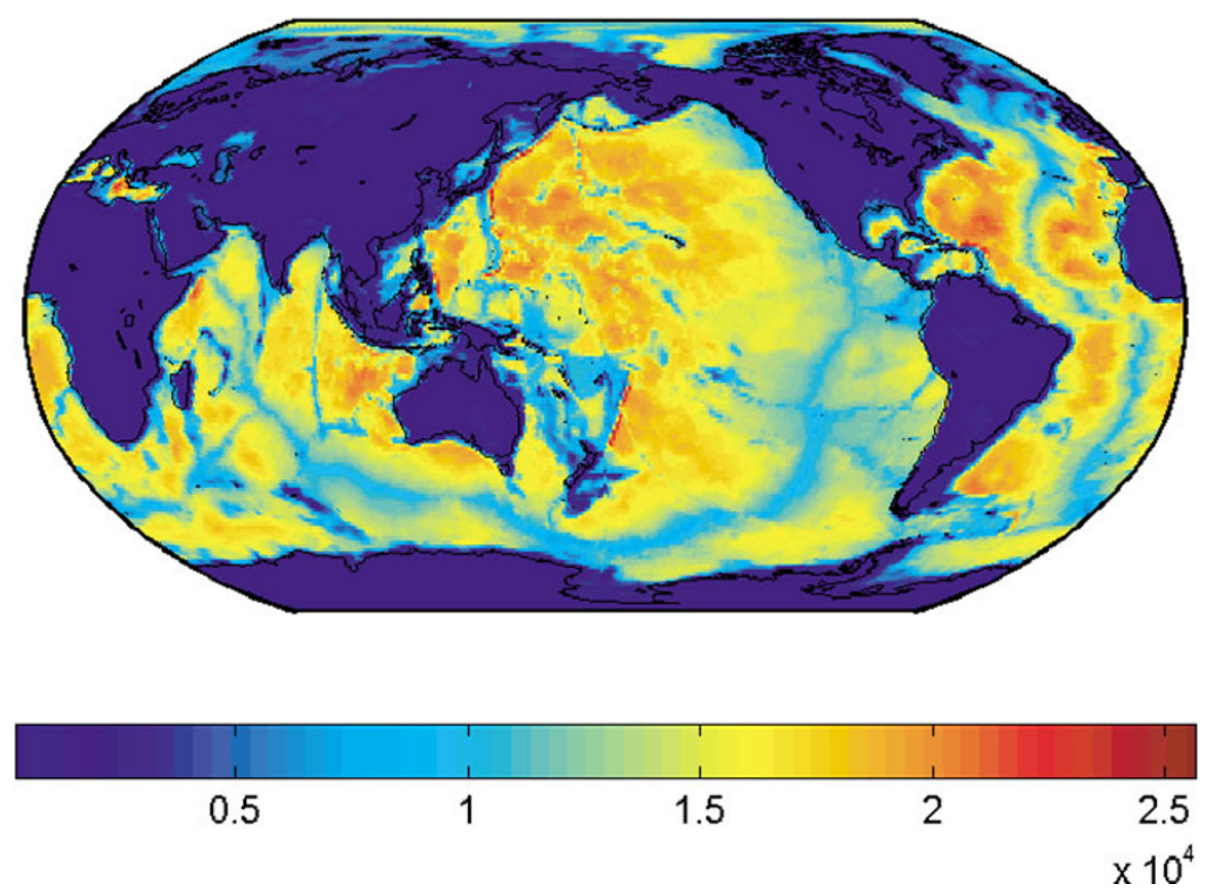

Fig. 1. Map of the adopted surface shell conductance (in S).
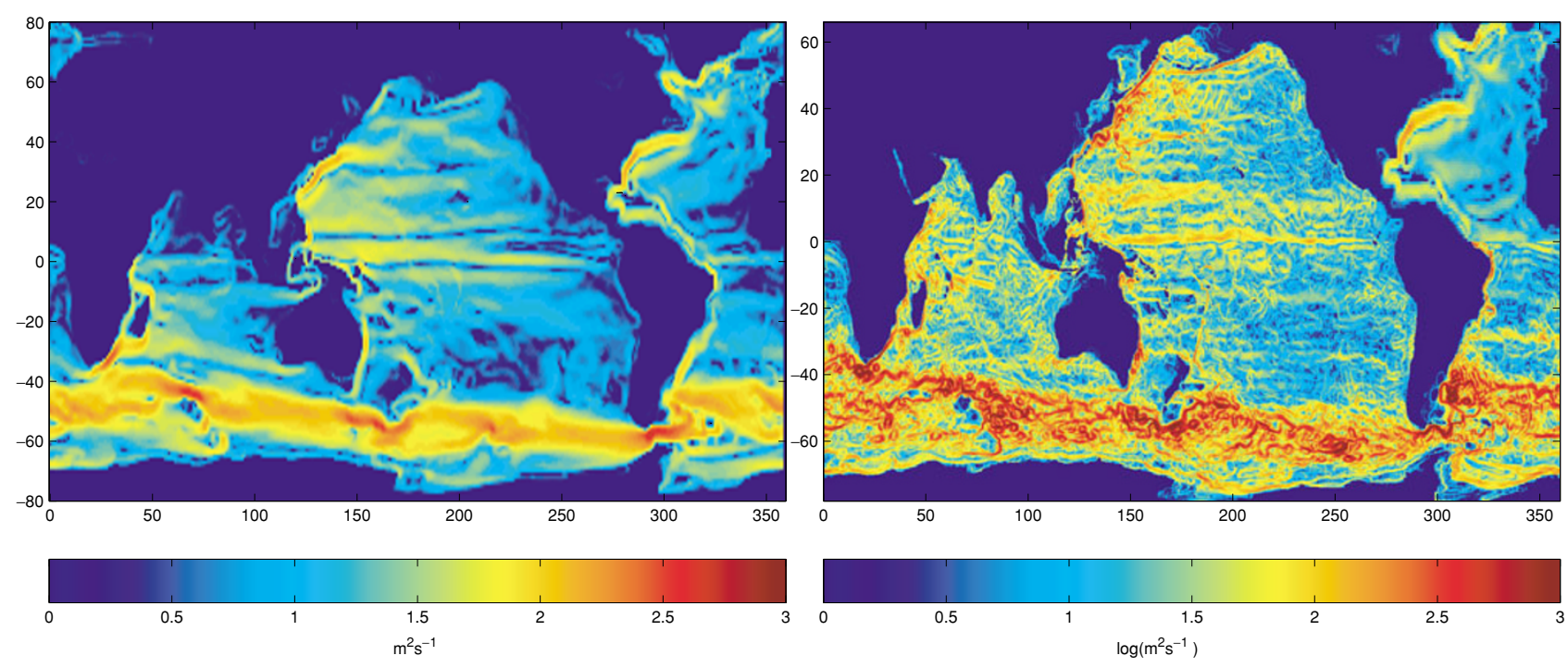

Fig. 2. Magnitude distribution of the ECCO (left panel) and OCCAM (right panel) velocity data. Magnitude is in $\log \left(\mathrm{m}^{2} \mathrm{~s}^{-1}\right)$.

from the static crustal field. Indeed, it may thus be possible to have an independent measure of ocean variability from satellite magnetometer records. The total range of $B_{r}$ variability for the ECCO 1 (1992-2002) simulation are given in the Fig. 5 for satellite altitudes. Strong variability of the signals can be seen over the Southern Indian and Australian Oceans $(0.5 \mathrm{nT})$. The high amplitude of the variability is due to the compounded effect of the ACC flow variability and its proximity to the southern geomagnetic pole. Smaller magnitude signals are also found in the Western Pacific and Northern Atlantic Oceans.

\subsection{Seasonal variation}

We estimated the mean flow velocities of Winter (January to March) and Summer (June to August) months for the year 2002 from ECCO output. The difference between
Summer and Winter flow velocity data (Fig. 6) shows strong variability along the equatorial region, possibly because the ocean flow responds vigorously to changes in the wind field at low latitudes. However changes of lesser extent can also be found over most of the other ocean areas.

As the amplitudes of fluctuations in the circulations are significantly lower than that of the mean circulation, the annual variation in the magnetic fields are quite different from the individual predictions. The difference between Summer and Winter predictions (Fig. 7) shows strong variability in the Indian Ocean, probably connected to the South Asian Monsoon. In addition, variations in the Western Boundary currents and ACC are also evident. The difference field has a range of $\pm 0.2 \mathrm{nT}$. 


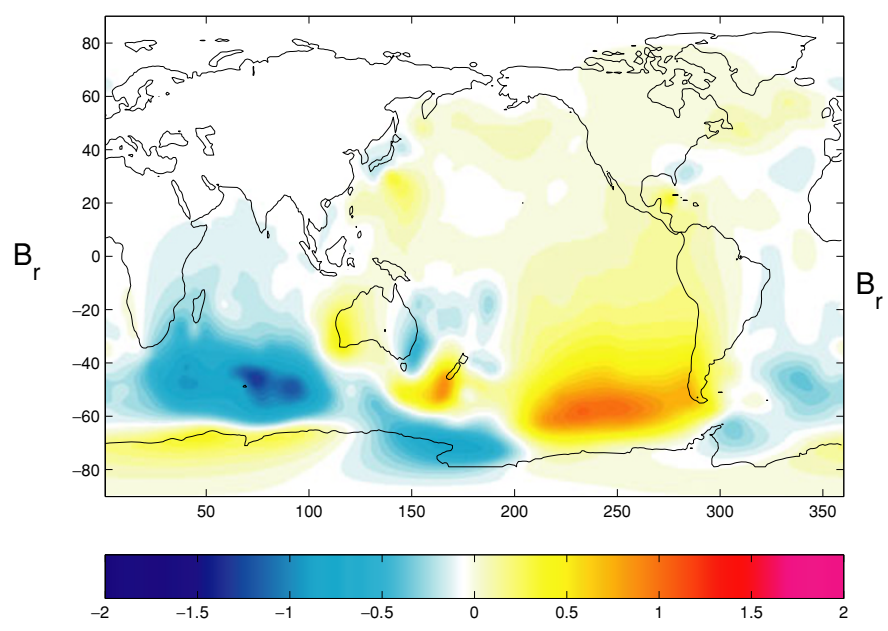

a)

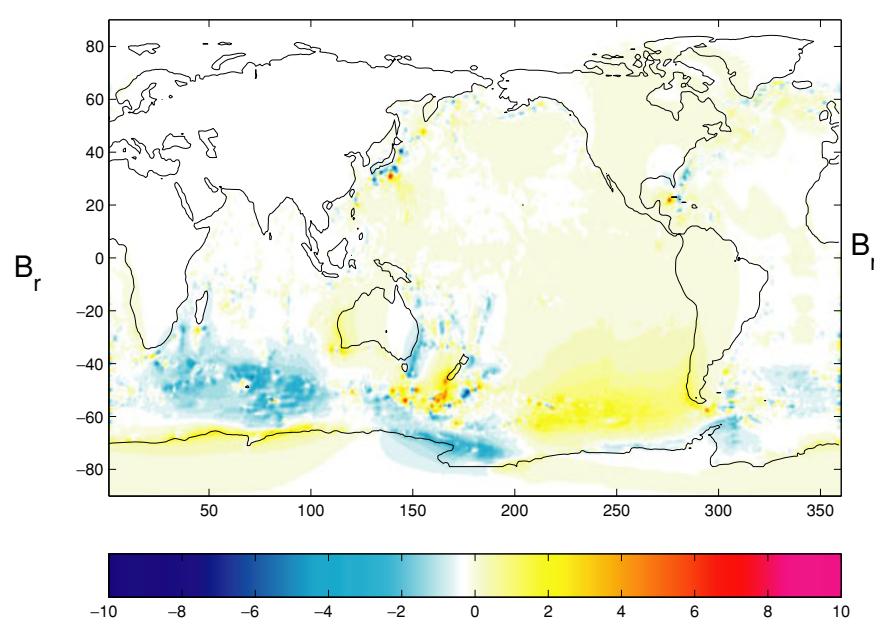

c)

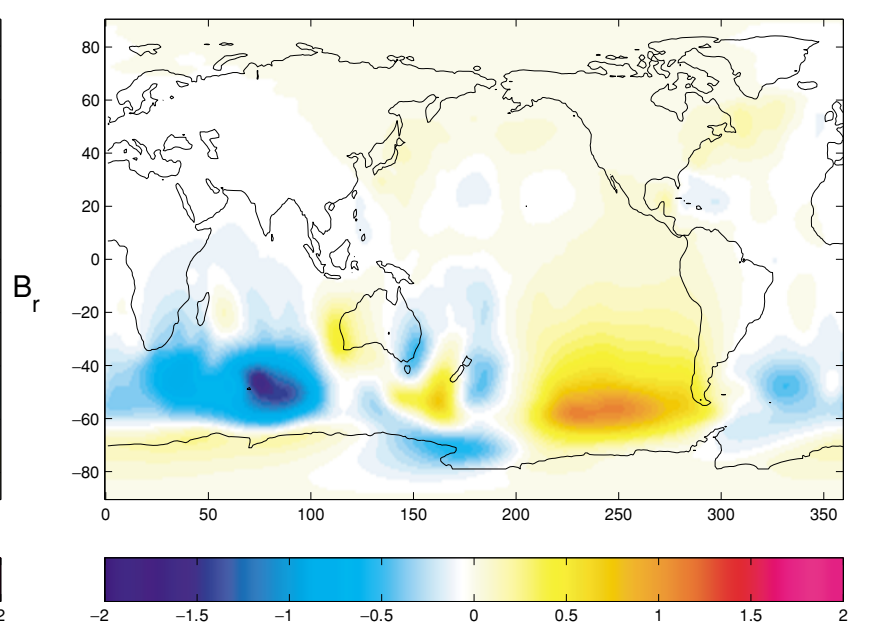

b)

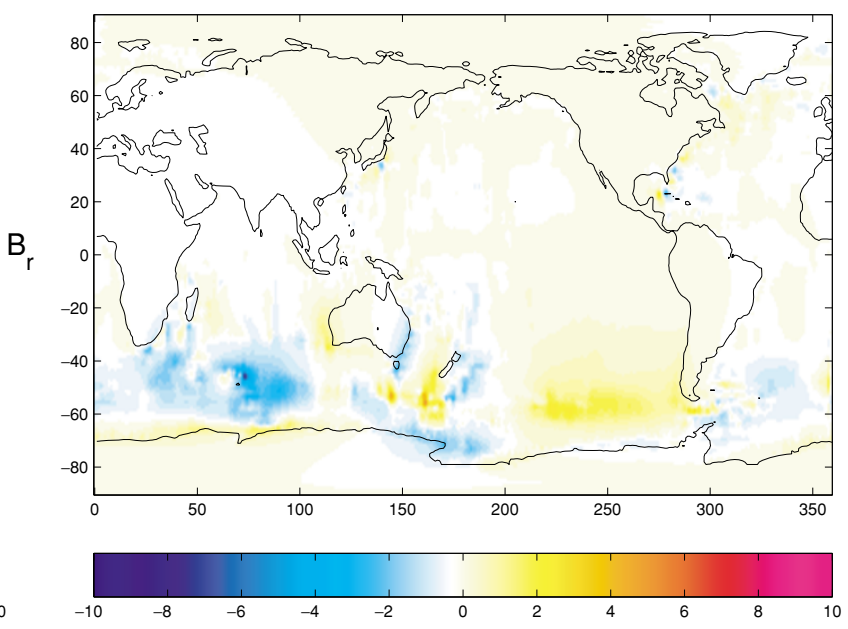

d)

Fig. 3. Comparison of simulations using OCCAM (left panels) and ECCO (right panels) results at sea level (bottom) and at $430 \mathrm{~km}$ above sea level (top) (in nT).

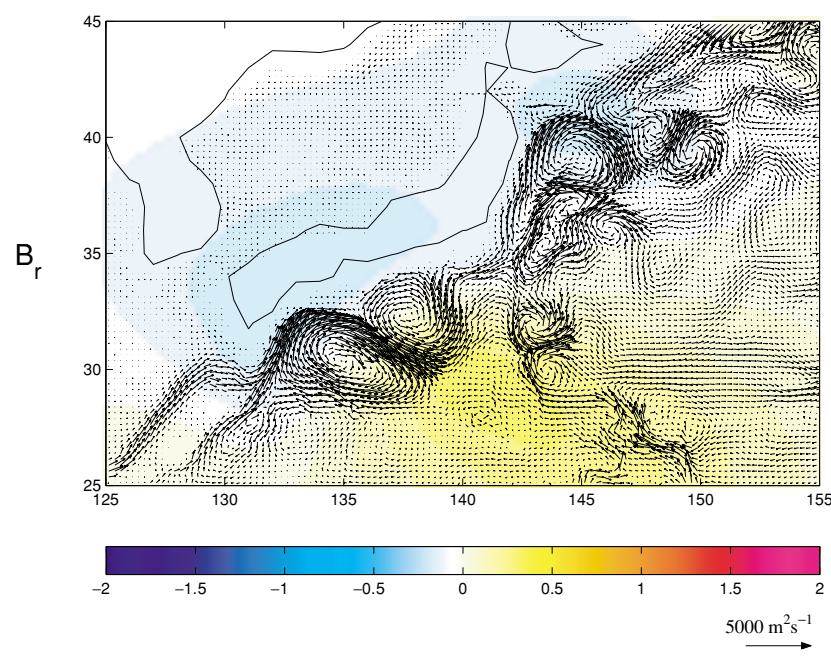

a)

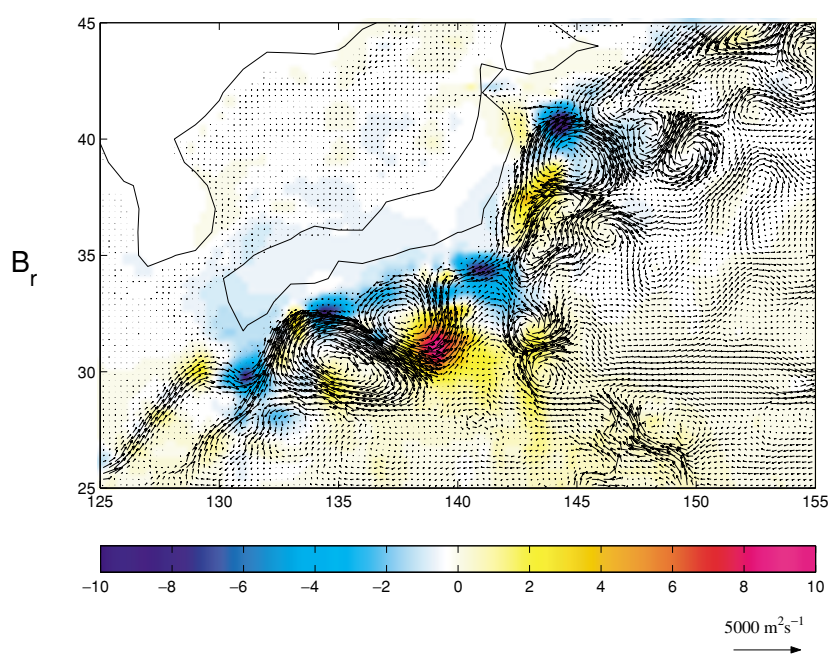

b)

Fig. 4. Predicted radial component of ocean induced magnetic field (in nT) for the Kuroshio Current region. (a) At Swarm altitude (430 km) and (b) at sea level.

\subsection{El Niño/Interannual variability}

The global oceanic circulation also exhibits interannual to interdecadal variability. Most prominent being the vari- ation associated with the El Niño and Southern Oscillation (ENSO). It is a change in the ocean-atmosphere system in the eastern Pacific which contributes to significant weather 


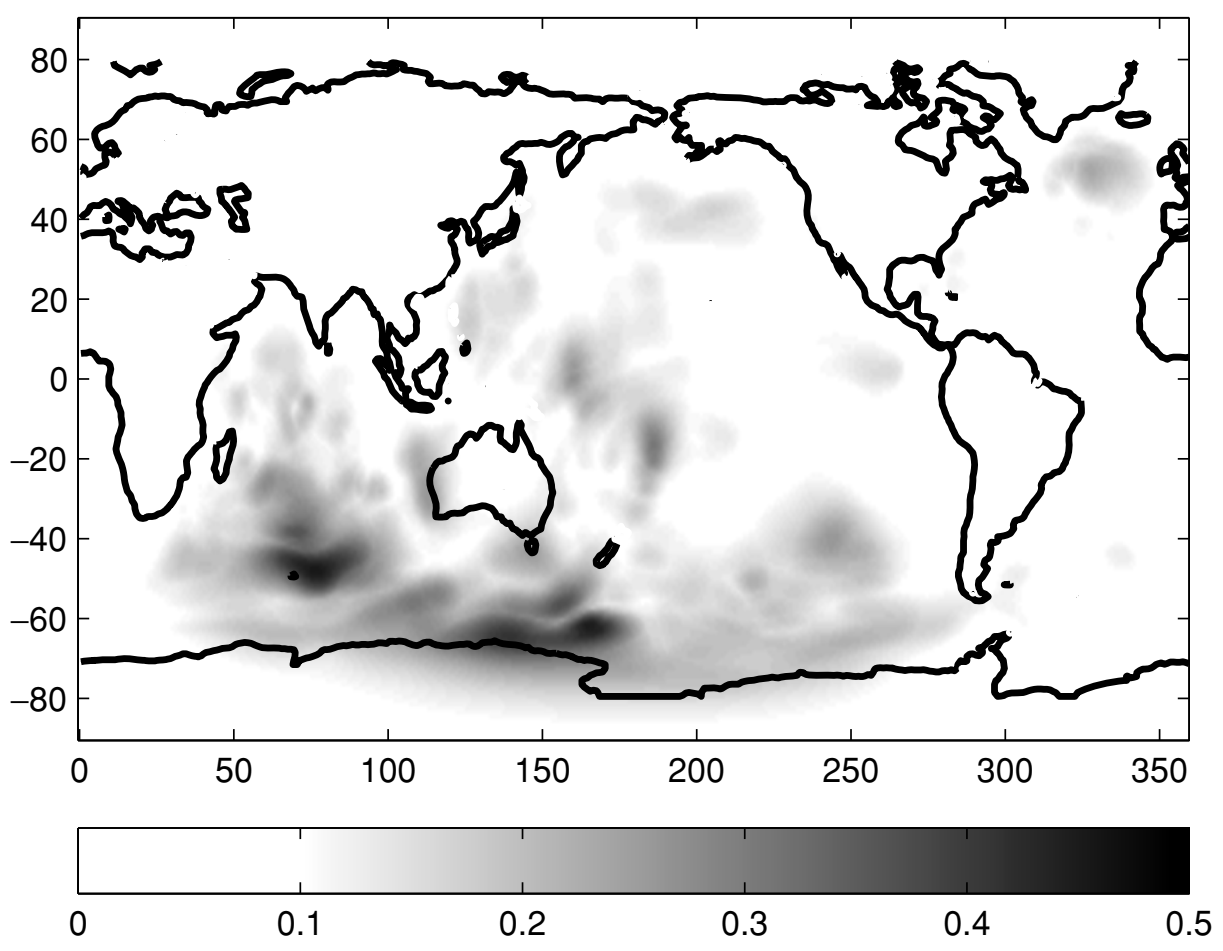

Fig. 5. The range of variability (in nT) of the simulations with ECCO 1 (1992-2002).

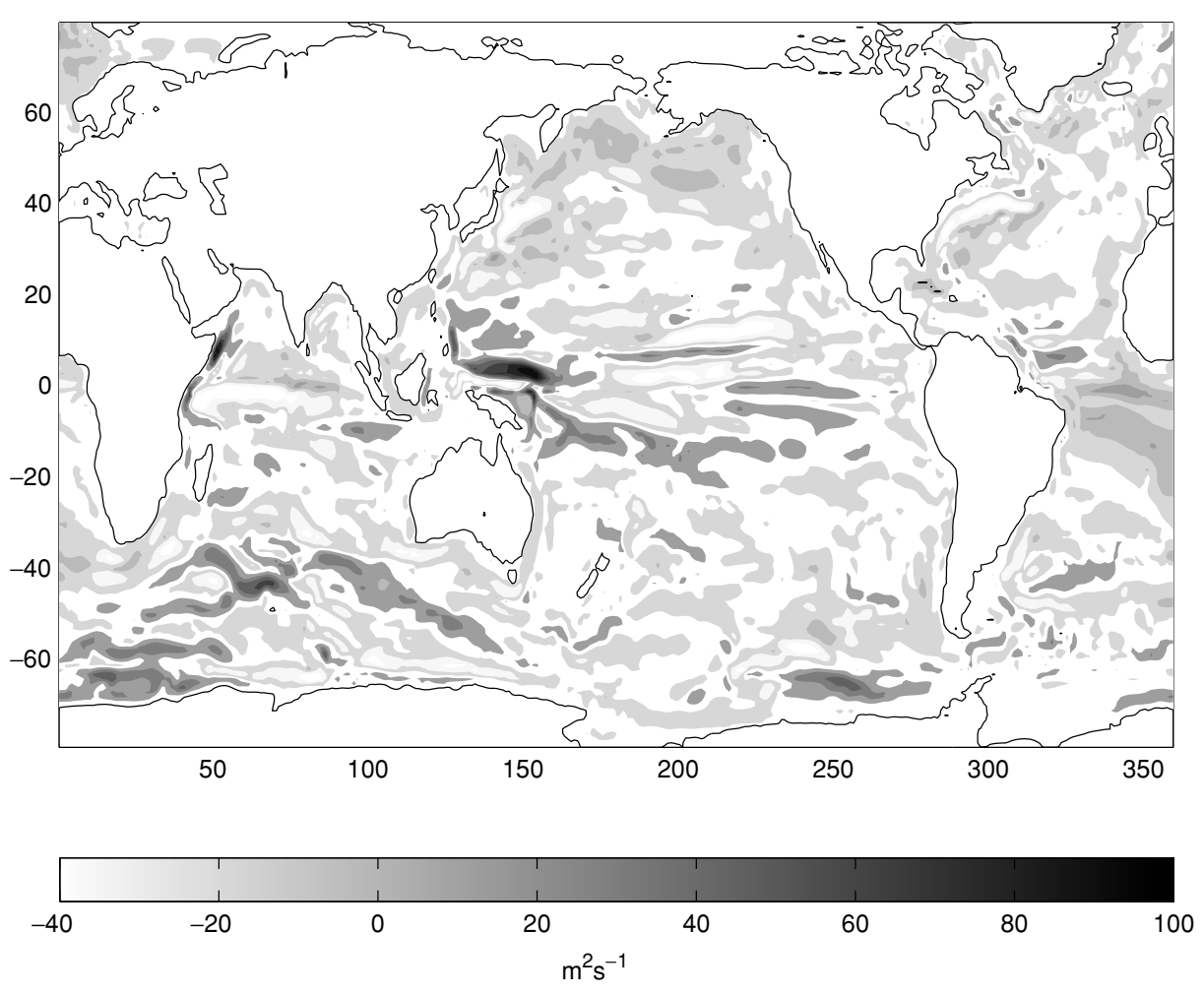

Fig. 6. Difference between Summer and Winter (2002) ocean velocity data. Units in $\mathrm{m}^{2} \mathrm{~s}^{-1}$.

changes around the world. In normal conditions, the trade winds blow towards the west and pile up warm water in the Western Pacific. During these conditions (called La Niña), the sea level is $0.5 \mathrm{~m}$ higher at Indonesia than at Ecuador. During El Niño, the trade winds relax allowing the warm equatorial current to flow towards the South Amer- ican coast. The recent El Niño event of 2002-2003 was weaker than the previous event during 1997-1998. We selected the El Niño during June 1997 to May 1998 for the simulations studies. Changed sea level gradient and relaxed trade winds reverse the surface currents along the equatorial region during an El Niño. Perhaps this is best illustrated by 


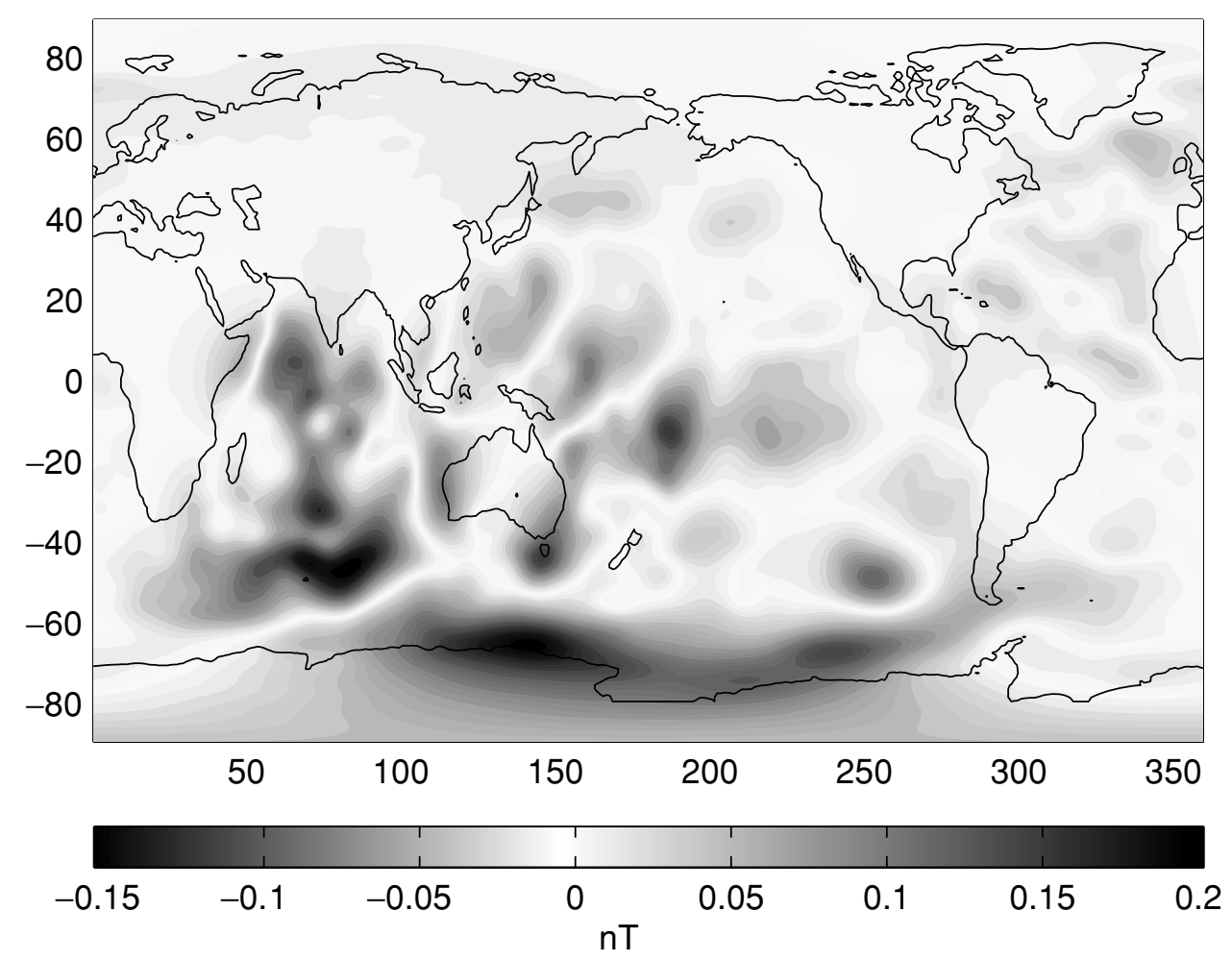

Fig. 7. Difference map $\left(\mathrm{B}_{r}\right)$ between Summer and Winter predictions (in $\mathrm{nT}$ ).
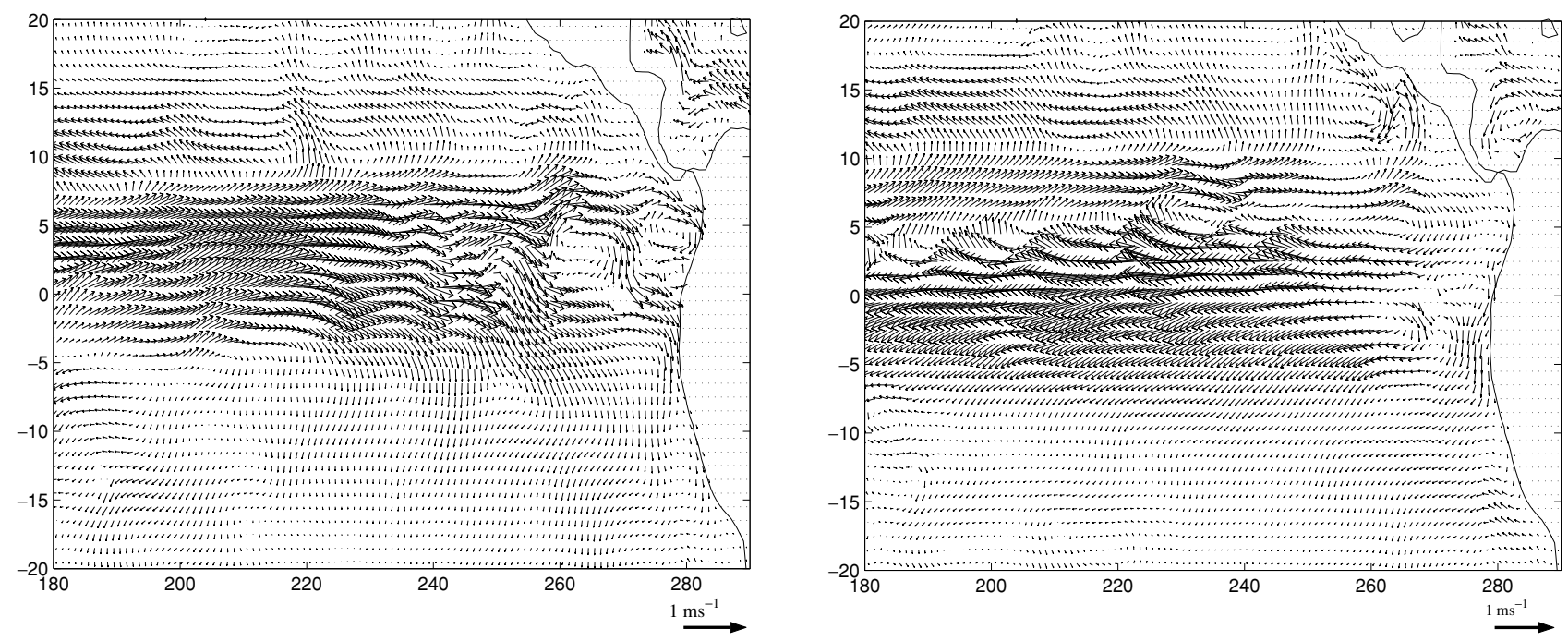

Fig. 8. Surface current (depth $<60 \mathrm{~m}$ ) velocity data from ECCO model November 1997 (El Niño) (left panel) and November 1999 (La Niña) (right panel). Units are in $\mathrm{ms}^{-1}$.

the surface velocity data from ECCO simulations as given in Fig. 8. The mean surface layer velocity vectors (depth down to $60 \mathrm{~m}$ ) for the November 1997 (El Niño) and the November 1999 (La Niña) periods show a dramatic reversal in flow direction. The depth extent of these currents is given in a stacked velocity plot (left panel of Fig. 9) for a rectangular area of latitude $0^{\circ}-5^{\circ}$ and longitude $200^{\circ}-212^{\circ}$. The influence of El Niño on the velocity seems to exist only in the upper 250 meters of the ocean. The velocities are within a range of $\pm 0.2 \mathrm{~m} / \mathrm{s}$ and have negligible magnitude for depth $>250 \mathrm{~m}$.

We averaged the circulation velocity data from June 1997 to May 1998 to represent the flow during the El Niño period. In addition, we computed the annual mean velocity for the year 1999 to represent the La Niña period. The predicted magnetic fields for the El Niño and La Niña periods are dominated by the gradients associated with ACC, with a weak amplitude range of $\pm 0.2 \mathrm{nT}$. The difference of these two predictions, although very weak, show an anomaly trending EW, as one would have expected from currents reversal. However this is probably dominated by velocity fluctuations associated with some other phenomena (right panel of the Fig. 9). In any case, the variability in the magnetic field has very low amplitude, as compared to seasonal 

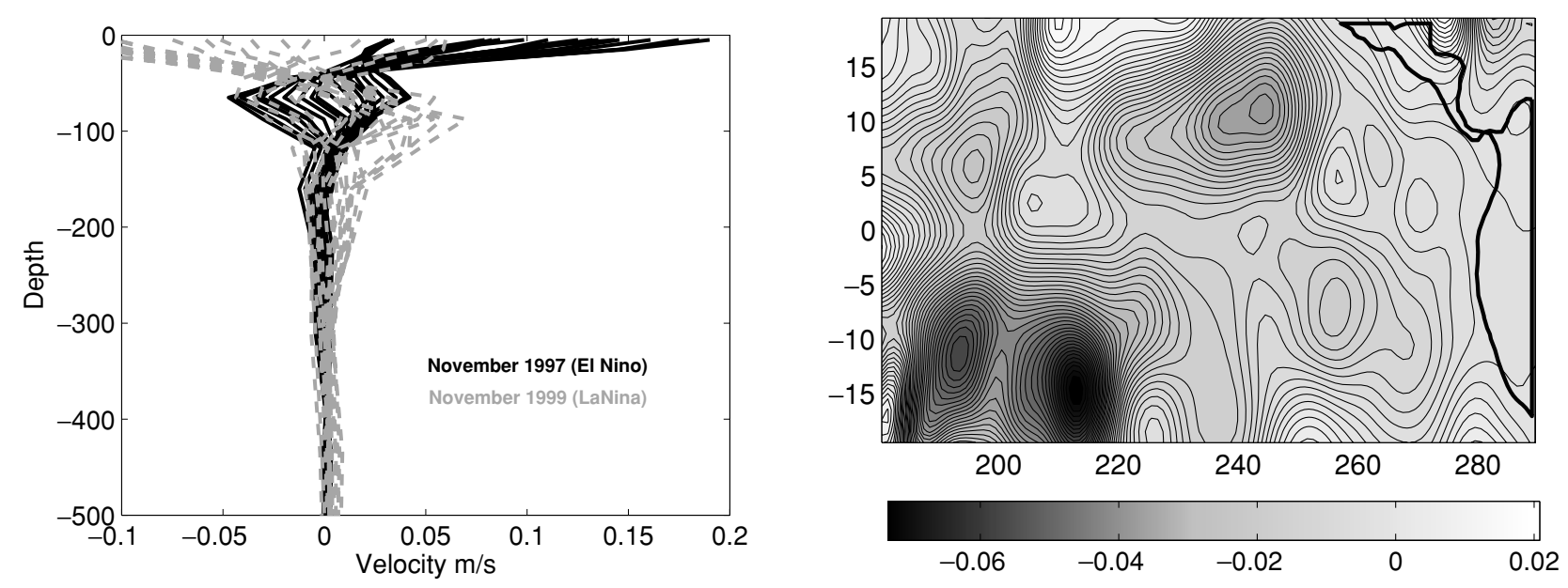

Fig. 9. Left panel: Stacked velocity (zonal) depth profiles for La Niña and El Niño periods (depth in m). Right panel: The difference of predicted magnetic fields between El Niño and La Niña periods (in nT).

variation. The reasons for such small variation could be: 1) The El Niño affects only a thin surface layer in the ocean, and the surface current reversal fails to produce appreciable changes in the depth integrated velocity, and 2) the low strength of the radial component of the Earth's magnetic field at low geomagnetic latitudes.

\section{Summary and Conclusion}

We predicted the magnetic field generated by ocean circulation using a global EM induction code based on the volume integral equation approach and considering a realistic $3 \mathrm{D}$ conductivity distribution for the Earth. The ocean circulation data were from two models viz. ECCO and OCCAM with $1^{\circ} \times 1^{\circ}$ resolution and $0.25^{\circ} \times 0.25^{\circ}$ resolutions, respectively. Magnetic fields predicted by both models have an amplitude range of $\pm 2 \mathrm{nT}$ at satellite altitude. These fields are predominantly influenced by the ACC and Western Boundary current. At satellite altitude the ocean model resolution does not have a strong influence on the pattern and amplitude of the magnetic signals. At sea level, however, the ECCO simulations have considerably less strength than those using OCCAM. A reason could be the presence of small scale circulation features such as ocean eddies in the OCCAM data. This is clearly visible along the Kuroshio current region where the OCCAM data produce an amplitude range of $\pm 10 \mathrm{nT}$. Nevertheless, effects of individual eddies are not evident when the magnetic fields are upwardly continued to Swarm altitude $(430 \mathrm{~km})$. Seasonal and interannual variations in the predicted magnetic fields were simulated by the data from the ECCO model. The difference between Summer and Winter data for the year 2002 has an amplitude range of $\pm 0.2 \mathrm{nT}$ at satellite altitude. An interesting region is the Indian Ocean. The variations in the magnetic field related to the surface current reversal during the 1997-1998 El Niño event produced considerably smaller amplitude than expected, probably due to its proximity to the geomagnetic equator. The small amplitude in the temporal magnetic variability seen in these results is in general agreement with previous studies (using OPYC and ECCO ocean model data) by J. Oberhuber and $\mathrm{R}$. Tyler which have been presented at international confer- ences (Tyler et al., 1998; Tyler, 2002, 2003). Because these three different studies involved three rather different parameterizations and numerical methods for calculating the magnetic fields from the flow there is now reasonable confidence that the small variability is not simply an artifact of either the numerical method or parameterizations.

Our simulations show a significant contribution of the ocean circulation generated magnetic signals at sea and satellite altitude. At sea level, the strong signals from the ocean eddies indicate the need of using higher resolution $\left(0.25^{\circ} \times 0.25^{\circ}\right.$ or less $)$ ocean circulation data to model the effects expected in ground based magnetometer data. At satellite altitude, the similar strength and global pattern of the magnetic signals predicted from two independent ocean circulation models also strengthens the case for being able to identify non-tidal ocean magnetic signals in future satellite data. Presently, however, the separation of these signals from the crustal magnetic fields in the satellite data may be difficult. Maus et al. (2005) showed the importance of correcting satellite data for tidal magnetic signals in crustal field modelling. Considering the non trivial contribution of the magnetic field from the ocean circulation, we propose to correct future crustal field anomaly maps by subtracting the predicted signals of the steady ocean circulation. As Swarm intends to bring out higher resolution crustal field anomaly map, this correction gains more importance. With the increasing precision in the modeling and measurement of geomagnetic fields, the identification of non-tidal ocean magnetic signals from satellite data and their use to gain information about global ocean water transport and their variations, stands as a realistic possibility.

Acknowledgments. ECCO and OCCAM ocean models were used to derive the ocean transport. ECCO is a contribution of the Consortium for Estimating the Circulation and Climate of the Ocean (ECCO) funded by the National Oceanographic Partnership Program, USA. The Swarm end-to-end simulation study was sponsored by the European Space Agency (ESA). One of the authors $(\mathrm{CM})$ acknowledges the permission of the Director, NGRI, Hyderbadad, India to publish this paper. Comments from Robert Tyler, Mike Purucker and Nils Olsen improved the original manuscript. 


\section{References}

Chave, A., On the theory of electromagnetic induction in the earth by ocean currents, J. Geophys. Res., 88, 3531-3542, 1983.

Everett, M., S. Constable, and C. Constable, Effects of near-surface conductance on global satellite induction responses, Geophys. J. Int., 153, 277-286, 2003.

Faraday, M., Experimental researches in electricity (Bakerian lecture), Philos. Trans. R. Soc. London, 122, 163-177, 1832.

Flosadóttir, A. H., J. C. Larsen, and J. T. Smith, Motional induction in North Atlantic circulation models, J. Geophys. Res., 102, 10,353$10,372,1997 \mathrm{a}$.

Flosadóttir, A. H., J. C. Larsen, and J. T. Smith, The relation of seafloor voltages to ocean transports in North Atlantic circulation models: Model results and practical considerations for transport monitoring, J. Physical Oceanography, 27, 1547-1565, 1997b.

Fofonoff, N. P., Physical properties of seawater: A new salinity scale and equation of state for seawater, J. Geophys. Res., 90, 3322-3342, 1985.

Junge, A., The telluric field in northern Germany induced by tidal motion in North Sea, Geophys. J. Int., 95, 523-533, 1988.

Kuvshinov, N. and N. Olsen, 3-D modelling of the magnetic fields due to ocean tidal flow, in Earth Observation with CHAMP. Results from Three Years in Orbit, edited by C. Reigber, H. Lühr, P. Schwintzer, and J. Wickert, pp. 359-366, Springer Verlag, 2005.

Kuvshinov, A. V., D. B. Avdeev, O. V. Pankratov, S. A. Golyshev, and N. Olsen, Modelling electromagnetic fields in 3D spherical Earth using fast integral equation approach, in $3 D$ Electromagnetics, edited by M. S. Zhdanov, and P. E. Wannamaker, chap. 3, pp. 43-54, Elsevier, Holland, 2002.

Kuvshinov, A. V., H. Utada, D. Avdeev, and T. Koyama, 3-D modelling and analysis of Dst C-responses in the North Pacific Ocean region, revisited, Geophys. J. Int., 160, 505-526, 2005.

Larsen, J. C., Electric and magnetic fields induced by deep see tides, Geophys. J. R. Astr. Soc., 16, 47-70, 1968.

Larsen, J. C. and T. Sanford, Florida Current volume transport from voltage measurements, Science, 227, 302-304, 1985.

Laske, G. and G. Masters, A global digital map of sediment thickness, EOS Trans. AGU, 78, F483, 1997.

Lilley, F., A. White, G. Heinson, and K. Procko, Seeking a seafloor magnetic signal from the Antarctic Circumpolar Current, Geophys. J. Int., 157, 175-186, 2004a.

Lilley, F., A. Hitchman, P. R. Milligan, and T. Pedersen, Sea-surface observations of the magnetic signals of ocean swells, Geophys. J. Int., 159, 565-572, 2004b.

Marshall, J., A. Adcroft, C. Hill, Perelman, and C. Heisey, A finite-volume, incompressible Navier-Stokes model for studies of the ocean on parallel computers, J. Geophys. Res., 102, 5753-5766, 1997.

Maus, S. and A. Kuvshinov, Ocean tidal signals in observatory and satellite magnetic measurements, Geophyc. Res. Lett, 31,
doi:10.1029/2004GC000, 634, 2004.

Maus, S., M. Rother, K. Hemant, H. Lühr, A. Kuvshinov, and N. Olsen, Earth's crustal magnetic field determined to spherical harmonic degree 90 from CHAMP satellite measurements, Geophys. J. Int., 164, 319330, doi:10.1111/j.1365-246X.2005.02833.x, 2006.

Palshin, N., L. Vanyan, I. Yegorov, and K. Lebedev, Electric field induced by the glodal ocean circulation, Physics Solid Earth, 35, 1028-1035, 1999.

Pankratov, O., A. Kuvshinov, and D. Avdeev, High-performance threedimensional electromagnetic modeling using modified Neumann series. Anisotropic case, J. Geomag. Geoelectr., 49, 1541-1547, 1997.

Sanford, T. B., Motionally induced electric and magnetic fields in the sea, J. Geophys. Res., 76, 3476-3492, 1971.

Schmucker, U., Electrical properties of the Earth's interior, in LandoltBörnstein, New-Series, 5/2b, pp. 370-397, Springer-Verlag, BerlinHeidelberg, 1985.

Singer, B., Method for solution of Maxwell's equations in non-uniform media, Geophys. J. Int., 120, 590-598, 1995.

Stephenson, D. and K. Bryan, Large-scale electric and magnetic fields generated by the oceans, J. Geophys. Res., 97, 15,467-15,480, 1992.

Tyler, R. H., Theoretical and Numerical Results on the Magnetic Fields Generated by Ocean Flow, EGS annual conference, 2002.

Tyler, R. H., Exploring and exploiting the magnetic fields generated by ocean flow, Geophysical Research Abstracts, 5, 2003.

Tyler, R., L. A. Mysak, and J. Oberhuber, Electromagnetic fields generated by a 3-D global ocean circulation, J. Geophys. Res., 102, 5531-5551, 1997.

Tyler, R. H., T. B. Sanford, and J. M. Oberhuber, Magnetic Fields Generated by Ocean Flow, AGU Fall Conference, 1998.

Tyler, R., J. Oberhuber, and T. Sanford, The potential for using ocean generated electromagnetic field to remotely sense ocean variability, Phys. Chem. Earth (A), 24, 429-432, 1999.

Tyler, R., S. Maus, and H. Lühr, Satellite observations of magnetic fields due to ocean tidal flow, Science, 299, 239-240, 2003.

Vivier, F., E. Maier-Reimer, and R. H. Tyler, Simulations of magnetic fields generated by the Antarctic Circumpolar Current at satellite altitude: Can geomagnetic measurements be used to monitor the flow?, Geophys. Res. Lett., 31, doi:10.1029/2004GL019, 804, 2004.

Webb, D. J., B. A. de Cuevas, and A. C. Coward, The first main run of the OCCAM global ocean model, Internal Document 34, Southampton Oceanography Centre, U.K., 1998.

Zhang, S.-L., GPBi-CG: generalized product-type methods based on BiCG for solving nonsymmetric linear systems, SIAM J. Sci. Comput., 18, 537-551, 1997.

C. Manoj (e-mail: manoj@gfz-potsdam.de), A. Kuvshinov, S. Maus, and H. Lühr 